\title{
Isotope Effect for the Penetration Depth in Superconductors
}

\author{
A. Bill, and V.Z. Kresin \\ Lawrence Berkeley Laboratory, University of California, Berkeley, CA 94720, USA
}

\author{
S.A. Wolf \\ Naval Research Laboratory, Washington D.C. 20375-5343
}

\begin{abstract}
We show that various factors can lead to an isotopic dependence of the penetration depth $\delta$. Nonadiabaticity (Jahn-Teller crossing) leads to the isotope effect of the charge carrier concentration $n$ and, consequently, of $\delta$ in doped superconductors such as the cuprates. A general equation relating the isotope coefficients of $T_{c}$ and of $\delta$ is presented for London superconductors. We further show that the presence of magnetic impurities or a proximity contact also lead to an isotopic dependence of $\delta$; the isotope coefficient turns out to be temperature dependent, $\beta(T)$, in these cases. The existence of the isotope effect for the penetration depth is predicted for conventional as well as for high-temperature superconductors. Various experiments are proposed and/or discussed.
\end{abstract}

\section{INTRODUCTION}

When speaking of the isotope effect (IE) in superconductors, one generally considers the influence of isotopic substitution on the superconducting critical temperature $T_{c}$. In systems where the pairing mechanism is at least partly mediated by the electron-phonon interaction $T_{c}$ depends on phonon energies. Thus, replacing one element by its isotope will affect $T_{c}$ via the change in phonon frequency. In a series of recent papers 13 we have shown that besides this most simple case, there are a number of other factors, not related to the pairing mechanism, that can strongly affect the isotope coefficient (IC) of $T_{c}$. The influence of the proximity effect, the presence of magnetic impurities and non-adiabaticity were specifically studied. Several experiments were proposed for conventional superconductors and the oxygen IE in high- $T_{c}$ materials was discussed. In the latter case it was experimentally shown (see e.g. Refs. 8 and references therein), that the value of the isotope coefficient of $T_{c}$ ranges from almost zero to values above 0.5 , depending on the doping process. The theory developed in Ref. 2 allowed us to present a systematic description of all experimental data collected to this day on the oxygen isotope effect of $T_{c}$ in $\mathrm{YBa}_{2} \mathrm{Cu}_{3} \mathrm{O}_{7-\delta}$ related systems. We stress here the fact, however, that the theory is not restricted to high- $T_{c}$ materials, but applies to conventional superconductors as well.

The influence on the IE of factors not related to the pairing mechanism 13 raises the question, whether besides $T_{c}$, there are other superconducting properties that also display these new effects. In the present paper we focus on one of the fundamental parameters, the penetration depth, and demonstrate that, indeed, it can display a non-trivial dependence on isotopic substitution. The treatment is based on our previous analysis in Ref. 2 that focused on the unconventional isotopic dependence of $T_{c}$. After isotope substitution in a superconductor $(M \rightarrow$
$M^{\star}=M+\Delta M, M$ is the ionic mass), the value of the critical temperature is shifted: $T_{c 0}^{\star}=T_{c 0}+\Delta T_{c 0}$. As is known, the isotope coefficient $\alpha_{0}$ is defined by the relation $T_{c 0} \sim M^{-\alpha_{0}}$ and is equal to $\alpha_{0}=-(M / \Delta M)\left(\Delta T_{c 0} / T_{c 0}\right)$. Suppose now that the superconductor is perturbed by some external factor as e.g. the presence of magnetic impurities or a normal film (proximity effect). Superconducting properties are affected by these factors. For example, $T_{c 0} \rightarrow T_{c}$ and, as a consequence, the dependency of the new critical temperature on the ionic mass $\left(T_{c} \sim M^{-\alpha}\right)$ will also be changed ( $\alpha \neq \alpha_{0}$, see Refs. 2. 3 ). This not only affects the isotope effect of $T_{c}$ but also, e.g., the IE of the penetration depth.

In the following, we are concerned with the isotopic dependence of the penetration depth. By analogy with $T_{c}$, we define the new isotope coefficient $\beta$ by the relation $\delta \sim M^{-\beta}$. Therefore, $\beta$ is determined by

$$
\beta=-\frac{M}{\Delta M} \frac{\Delta \delta}{\delta}
$$

where $\Delta \delta$ is the shift of the penetration depth induced by isotopic substitution.

The structure of the paper is as follows. Section II is concerned with the non-adiabatic IE, whereas Secs. III] and IV] address the impact of magnetic scattering and the proximity effect on the IC, respectively. An interesting property of the two latter factors affecting the IE is that the isotope coefficient appears to be temperature dependent. We not only demonstrate here that such effects exist but that they are also sizeable. Moreover, we argue that the measure of the IC can be used to probe the presence of non-adiabaticity, magnetic impurities or the proximity effect in superconductors. The theory leads us to propose several experiments for conventional and high-temperature superconductors. We also discuss hgur recent experiments done on high- $T_{c}$ superconductors can be described by our theory. 


\section{ISOTOPE EFFECT OF $\delta$ AND NON-ADIABATICITY}

The theory for the non-adiabatic IE of $T_{c}$ has recently been proposed 1 and applied to describe the oxygen IC in high-temperature superconductorst 3 as well as properties of manganites 10 . We show in this section that other quantities like the penetration depth $\delta$ of a magnetic field can be affected by isotopic substitution through this nonadiabatic channel.

\section{A. Theory}

\section{Isotope effect of $n$}

Let us describe the main concept of the non-adiabatic IE introduced in Ref. 11 (see also Ref. 2). Consider a system composed of a conducting system and a charge reservoir (as for example the $\mathrm{CuO}_{2}$ plane and the buffer layer of a cuprate high- $T_{c}$ superconductor) and assume that there is a charge transfer between these subsystems. In general, the reservoir-conducting-layer charge-transfer process occurs through a group of atoms located in both subsystems and/or bridging the two. For example, the charge transfer between the $\mathrm{CuO}_{2}$ plane and the reservoir ( $\mathrm{CuO}$ chain for $\mathrm{YBCO}, \mathrm{BiO}$ plane for Bi-based oxides, etc...) occurs through the apical oxygen. The electronic degeneracy and the corresponding Jahn-Teller instability of the group of atoms leads to the crossing of electronic terms. Because of the breakdown of the adiabatic approximation for these atoms, the electronic and ionic degrees of freedom can no longer be considered as decoupled. The potential energy surface considered as a function of the ionic coordinate is composed of two electronic terms with two close minima. For example, the motion of the apical oxygen which is a lighter ion of the $\mathrm{Cu}-\mathrm{O}-\mathrm{Cu}$ Jahn-Teller complex is characterized by two minima (a more detailed discussion is given in Refs. 11.2; experimental evidence of these minima can be found in Refs. 11, 12, Note that each of these terms can be considered in the harmonic approximation. The "double-well" is thus not related to the anharmonicity of the lattice, but to the non-adiabatic behaviour of the group of ions involved in the charge-transfer process. In a qualitative picture, the charge transfer between the conducting and the reservoir subsystems will involve the tunneling of the ionic complex between the two minima and, as a consequence, the density of charge carriers in the conducting subsystem will depend on the ionic masses. Properties depending on the charge-carrier density $n$ will thus be ionic mass dependent $(n \equiv n(M))$ and exhibit an isotope effect.

In order to consider the Jahn-Teller crossing (where the adiabatic approximation is not applicable) it is convenient to use the so-called diabatic representation (see, e.g., Ref. 14). In this representation one can show that the energy level splitting has the form

$$
H_{12}=<\Psi_{1}\left|H_{e}\right| \Psi_{2}>\simeq L_{0} F_{12}
$$

with

$$
\begin{aligned}
L_{0} & =\int \mathrm{d} \mathbf{r} \psi_{1}^{*}\left(\mathbf{r}, \mathbf{R}_{\mathbf{0}}\right) H_{e} \psi_{2}\left(\mathbf{r}, \mathbf{R}_{\mathbf{0}}\right), \\
F_{12} & =\int \mathrm{d} \mathbf{R} \Phi_{1}^{*}(\mathbf{R}) \Phi_{2}(\mathbf{R})=F_{12}(M)
\end{aligned}
$$

$H_{e}$ is the electronic part of the Hamiltonian and $\Psi_{j}(\mathbf{r}, \mathbf{R})=\psi_{j}(\mathbf{r}, \mathbf{R}) \Phi_{j}(\mathbf{R})(j=1,2) . \quad \psi_{j}(\mathbf{r}, \mathbf{R})$ is the electronic wave function (with electronic coordinates $\mathbf{r}$ ) depending parametrically on the nuclear coordinates $\mathbf{R}$ and $\Phi_{j}(\mathbf{R})$ is the vibrational wave functions. The last equality in Eq. (2) was obtained because $\psi_{j}(j=1,2)$ is a slow varying function of $\mathbf{R}$ and can thus be evaluated at $\mathbf{R}_{0}$, the crossing of electronic terms. $L_{0}$ does not depend on the ionic masses. On the other hand, the important Franck-Condon factor $F_{12}$ depends on the lattice wave functions $\Phi_{j}$ and thus on ionic masses $M$.

The reservoir-conducting-layer charge transfer is accompanied by a change of electronic terms and can be visualized as a multi-step process. First, the charge carrier can move from the reservoir to the group of ions (e.g. from the chains to the apical oxygen in YBCO). Then, the complex tunnels to the other electronic term $\left(\Psi_{1} \rightarrow \Psi_{2}\right)$. As a final step, the charge carrier can hop to the conducting layer. We emphasize that this charge transfer is a dynamical process and leads to a stationary state in the sense that the charges oscillate in time between the reservoir and the conducting subsystems, with a frequency given by Eq. (2). Since the ionic masses affect $F_{12}$, and thus $H_{12}$, different isotopic masses imply different values of the characteristic charge-transfer frequency. This in turn affects the charge-carrier concentration $n$.

The isotope coefficient of $T_{c}$ can be written as $\alpha=$ $\alpha_{p h}+\alpha_{n a}$, where $\alpha_{p h}=\left(M / T_{c}\right)\left(\partial T_{c} / \partial \Omega\right)(\partial \Omega / \partial M)$ is the usual (BCS) phonon contribution $(\Omega$ is a characteristic phonon energy) and the non-adiabatic contribution is given by:

$$
\alpha_{n a}=\gamma \frac{n}{T_{c}} \frac{\partial T_{c}}{\partial n}
$$

where the parameter $\gamma=-M / n(\partial n / \partial M)$ has a weak logarithmic dependence on $M$ (see Ref. 1). Eq. (4) shows that the IC of $T_{c}$ depends on the doping of the conducting layer and on the relation $T_{c}(n)$. This result was used in Ref. 2 to analyse the IE of high-temperature superconductors.

\section{Non-adiabatic isotope effect of $\delta$}

Let us now focus on the non-adiabatic IE of the penetration depth. As shown above (see also Refs. 1, 2, , nonadiabaticity for doped materials such as cuprates, leads to the dependence of the charge-carrier concentration $n$ 
on the ionic mass $M$, that is $n=n(M)$. In the weakcoupling London limit the penetration depth is given by the well-known relation

$$
\delta^{2}=\frac{m c^{2}}{4 \pi n_{s} e^{2}}=\frac{m c^{2}}{4 \pi n \varphi\left(T / T_{c}\right) e^{2}} \quad .
$$

where $m$ is the effective mass. $n_{s}$ is the superconducting density of charge carriers, related to the normal density $n$ through $n_{s}=n \varphi\left(T / T_{c}\right)$. The function $\varphi\left(T / T_{c}\right)$ is a universal function of $T / T_{c}$. For example, for conventional superconductors, $\varphi \simeq 1-\left(T / T_{c}\right)^{4}$ near $T_{c}$, whereas $\varphi \simeq 1$ near $T=0$ (in the absence of magnetic impurities; their influence is discussed at the end of this and in the following sections). Note that the relation $\delta^{-2} \sim n_{s}$ is also valid in the strong-coupling case (see, e.g., Refs. 3).

We can now determine the isotope coefficient $\beta$ of the penetration depth from the relation (5):

$$
\beta \equiv-\frac{M}{\delta} \frac{\partial \delta}{\partial n_{s}} \frac{\partial n_{s}}{\partial M}=\frac{M}{2 n_{s}} \frac{\partial n_{s}}{\partial M} \quad .
$$

Because of the relation $n_{s}=n \varphi(T)$, one has to distinguish two contributions to $\beta$. There is a usual (BCS) contribution, $\beta_{p h}$, arising from the fact that $\varphi\left(T / T_{c}\right)$ depends on ionic mass through the dependency of $T_{c}$ on the characteristic phonon frequency. Indeed, isotopic substitution leads to a shift in $T_{c}$ and thus in $\delta$, which might be noticeable near $T_{c}$ (see the discussion in Sec. [IIB). In this paper, however, we focus on the non-trivial manifestation of isotopic substitution, arising from the isotope dependence of the charge-carrier concentration $n$.

From Eq. (5) and the relation $n_{s}=n \varphi(T)$ it follows that

$$
\beta=\beta_{n a}+\beta_{p h}
$$

where

$$
\begin{gathered}
\beta_{n a}=\frac{M}{2 n} \frac{\partial n}{\partial M} \\
\beta_{p h}=\frac{M}{2 \varphi(T)} \frac{\partial \varphi(T)}{\partial M}
\end{gathered}
$$

Note that $n(M)$ is the normal-state charge-carrier concentration. Thus, unlike $\beta_{p h}$ (which depends on $\Phi\left(T / T_{c}\right)$ ), the non-adiabatic coefficient $\beta_{n a}$ does not depend on parameters characterizing the superconducting state. This effect should be observed near $T=0 \mathrm{~K}$, where $\beta_{p h}$ is negligibly small.

Comparing Eq. (4) and Eq. (8) one infers that $\beta_{n a}=$ $-\gamma / 2$ and thus establish a relation between the nonadiabatic isotope coefficients of $T_{c}$ and $\delta$ :

$$
\alpha_{n a}=-2 \beta_{n a} \frac{n}{T_{c}} \frac{\partial T_{c}}{\partial n}
$$

This result holds for London superconductors. The equation contains only measurable quantities and can thus be verified experimentally. It is interesting to note that $\beta_{n a}$ and $\alpha_{n a}$ have opposite signs when $\partial T_{c} / \partial n>0$ (which corresponds to the underdoped region in high- $T_{c}$ materials).

In later sections we will discuss the influence of magnetic impurities on the isotope effect of $T_{c}$ and $\delta$. We note here that in the presence of magnetic impurities the relation $n_{s}=n \varphi(T)$ remains valid, but $\varphi(T)$ depends now on the direct scattering amplitude $\Gamma_{2}$ defined in Sec. III B (this results, e.g., in the inequality $n_{s}(T=0)<n$ in the gapless regime). As a consequence, magnetic impurities affect $\beta_{p h}$ in Eq. (9), but leave $\beta_{n a}$ and thus Eq. (10) unchanged.

\section{B. Non-adiabatic isotope effect in high- $T_{c}$ superconductors}

The value of the parameter $\gamma$ (see Eq. (位) has been evaluated in Ref. 2 for Pr-substituted (YPrBCO) and oxygen-depleted (YBCO) $\mathrm{YBa}_{2} \mathrm{Cu}_{3} \mathrm{O}_{7-\delta}$ and was found to be $\gamma=0.16$ and $\gamma=0.28$ respectively. With Eq. (10) one then obtains the IC of the penetration depth $\beta_{n a}=-0.08$ in the first case and $\beta_{n a}=-0.14$ for the latter.

Experiments have been performed on $\mathrm{YBCO}$ and $\mathrm{La}_{2-x} \mathrm{Sr}_{x} \mathrm{CuO}_{4} \mathrm{O}$ (LSCO) which indicate that the Meissner fraction (and thus the penetration depth) indeed displays an isotope shift. Unfortunately, the measurements on YBCO have been done near $T_{c}$ where any superconductor (conventional or high- $T_{c}$ ) displays an isotope shift of $\delta$ through the dependency $\delta\left(T / T_{c}\right) \sim \sqrt{1-T^{4} / T_{c}^{4}}$ and corresponds thus to the usual BCS IE (this is the contribution $\beta_{p h}$ arising through $\varphi(T)$ as discussed in the last section; see also Ref. 15). The BCS isotope coefficient of $\delta$ (near $T_{c}$ ) can easily reach values of the order observed in the experiment 6 , even for a very small IC of $T_{c}$ (with a value $\alpha=0.025$ as observed in $\mathrm{YBa}_{2} \mathrm{Cu}_{3} \mathrm{O}_{7-\delta}$ one obtains $\beta \sim-0.6$ for $T / T_{c} \sim 0.95$ ). To avoid the BCS contribution to the IC that appears in all superconductors because of the strong dependency $\delta\left(T / T_{c}\right)$ near $T_{c}$ it is better to study experimentally the IE of the penetration depth near $T=0$. This statement is valid if one is interested in determining the non-adiabatic contribution to the IC $\beta$ but is not general. As will be shown below, it is for example possible to extract the influence of magnetic impurities on the IE even near $T_{c}$.

The situation with the measurements of Ref. 9 is different. The experiments 9 done on LSCO were obtained near $T=0$ and reflect the unconventional dependence $\delta(M)$ (the BCS contribution to the IC of $\delta$ vanishes at these temperatures). The shift has been measured near $x \simeq 0.11$ and $x \simeq 0.15$.

Near the concentration $x \simeq 0.11, T_{c}$ eriences a small depression as a function of doping $x .16$ Although several explanations have been proposed (as, e.g., the presence of electronic inhomogeneities, a change of the electronic density of states due to lattice distortions, im- 
purity scattering, magnetic ordering, etc..17) the presence of the dip is not well understood yet. The choice of this concentration is thus unfortunate and inappropriate for the study of the IE. It was argued that there is no influence of isotopic substitution on the charge carrier concentration $n$ near $x \simeq 0.11$ and that $n$ can therefore not depend on the ionic mass. To support this idea the influence of isotopic substitution on the structural (tetragonal to orthorhombic) transition temperature $T_{s}$ was studied 8 . The assertion is based on the assumption that $T_{s}$ depends solely on $n$, that there is a monotonic relation between $T_{s}$ and $n$ with non-zero slope and a one-to-one correspondance between $m_{\text {and }} x$. However, the experiments performed on LSCO 17 show that the hole concentration $n$ dependency of $T_{c}$ and $T_{s}$ as well as the relation $n(x)$ are not well established, especially in the region of the dip. The structural phase transition temperature $T_{s}$ has also only been measured at three points in the vicinity of the dip 16, 17, 9. Since $T_{c}$ has an unexpected behaviour it would be of interest to study the detailed dependency $T_{s}(x)$ in this region. Furthermore, as known from other high-temperature superconductors (as, e.g., YBCO) it is unlikely that the relation between $n$ and $x$ is linear. We emphasize that our analysis of the IC from Eqs. (4) and (10) does not require any assumption on the relation $n(x)$ since we rely solely on the experimental relation as obtained, e.g., from $\mu \mathrm{SR}$ experiments (in this case one has $T_{c}\left(\sigma \sim n_{s} / m^{\star}\right)$; see Ref. 3). In short, since the structural and electronic properties are not well understood in the region around $x \simeq 0.11$, it does not allow a conclusive statement on the IE of $\delta$.

The only experimental result 9 that can be discussed, and that does not contain the BCS contribution is the one done at $x \simeq 0.15$ (for LSCO). Using Eq. (11) and the value of the experimentally observed relative shift $\Delta \delta / \delta=2 \%$ one obtains $\beta_{n a} \simeq 0.16$ [and $\gamma=0.32$, cf. Eq. (4) and (10)] which is in good agreement with the calculations presented above. Indeed, it is of the same order as $\beta_{n a}$ for oxygen-depleted YBCO. This is consistent with the fact that in both these materials the reservoir$\mathrm{CuO}_{2}$-plane charge transfer involves the same group of ions including the apical oxygen. The larger value for LSCO can be traced back to the fact that in YBCO one has taken into consideration the presence of magnetic impurities (which also enhances the IC), whereas in LSCO there is no indication of the presence of significant quantities of such impurities $\mathrm{g} c$

One notes further that the IC of $\delta$ is much larger in YBCO and LSCO than in YPrBCO. The reason for this discrepancy can be twofold. On the one hand, the chargetransfer channel is different in YPrBCO from the two other materials 2 (e.g. it does not involve the apical oxygen). On the other hand, the concentration of magnetic impurities is higher in YPrBCO than in YBCO (and LSCO). This was taken into account in the calculation of $\alpha_{n a}$ and influences the value of $\beta_{n a}$ in YBCO related systems as well. A larger part of the IC is thus due to the magnetic impurities in YPrBCO (see Ref. 2 for a detailed study of the interplay between non-adiabaticity and magnetic impurities).

One should add that the experiment determining the IC through magnetic susceptibility measurement 8 is rather inaccurate. More reliable data could be obtained by measuring the penetration depth shift using microwave measurements or the Josephson effect. It would be interesting to perform experiments on the systems discussed above that display the non-adiabatic chargetransfer channel.

As stressed earlier and according to the analysis of Ref. 2 one has, however, to include the contribution of magnetic impurities to describe correctly the IE of $T_{c}$ in some of the high- $T_{c}$ superconductors. The magnetic impurities also directly affect the IC of $\delta$ and should be treated as well. This will be done in the next section.

\section{ISOTOPE EFFECT OF $\delta$ AND MAGNETIC IMPURITIES}

As described in Refs. 2, 3 magnetic impurities modify the IC of $T_{c}$. We show here that it can also induce an IE of the penetration depth. Consider a superconductor (conventional or high $T_{c}$ ) doped with magnetic impurities. Abrikosov and Gor'kov 18 have shown that these impurities act as pair breakers. In the dirty limit one can calculate the penetration depth analytically for temperatures near $T_{c}$ and at $T=0$.

\section{A. Magnetic impurity contribution to $\beta$ near $T_{c}$}

The penetration depth $\delta$ in the presence of magnetic impurities and near $T_{c}$ has been calculated by Skalski et al. 19 . Taking terms up to the order $\Delta^{2}$ the result is given by 19 .

$$
\delta^{-2}=\sigma \frac{\Delta^{2}(T)}{T_{c}} \zeta\left(2, \gamma_{s}+\frac{1}{2}\right)
$$

where $\sigma=4 \sigma_{N} / c$ ( $\sigma_{N}$ is the normal state conductivity), $\zeta(z, q)=\sum_{n \geq 0} 1 /(n+q)^{z}$ and $\gamma_{s}=\Gamma_{s} / 2 \pi T_{c}\left(\Gamma_{s}=\tilde{\Gamma}_{s} n_{M}\right.$ is the spin-flip scattering amplitude; $n_{M}$ is the concentration of magnetic impurities and $\tilde{\Gamma}_{s}$ is a constant). Near $T_{c}$, the order parameter can be written as $1 \mathrm{~g}$ :

$$
\Delta^{2}=2 \Gamma_{s}^{2}(1-\tau) \frac{1-\bar{\zeta}_{2}+(1-\tau)\left[\frac{1}{2}-\bar{\zeta}_{2}+\bar{\zeta}_{3}\right]}{\bar{\zeta}_{3}-\bar{\zeta}_{4}} \equiv 2 \Gamma_{s}^{2} \frac{N_{1}}{D_{1}}
$$

with $\bar{\zeta}_{z}=\gamma_{s}^{z-1} \zeta\left(z, \gamma_{s}+1 / 2\right), z=1,2, \ldots$ and $\tau=T / T_{c}$. Using Eqs. (11) and (12) one can calculate the isotope coefficient of the penetration depth in the presence of magnetic impurities $\left(\beta_{m}\right)$. In order to single out the impact of magnetic impurities on the value of the IC, we calculate the quantity $\tilde{\beta}_{m}=-(M / \Delta M)(\Delta \tilde{\delta} / \tilde{\delta})$ [cf. Eq. (11)], where $\tilde{\delta}=\delta\left(T, \Gamma_{s}\right) / \delta(T, 0)$. As a result, the IC is written 
as a difference $\tilde{\beta}_{m}=\beta_{m}-\beta_{0}$ where $\beta_{m}\left(\beta_{0}\right)$ is the isotope coefficient of $\delta\left(T, \Gamma_{s}\right)(\delta(T, 0))$. Using the relation $(\partial / \partial q) \zeta(z, q)=-z \zeta(z+1, q)$ one can express $\tilde{\beta}_{m}$ in the form:

$$
\tilde{\beta}_{m}=\left(R_{1}-R_{0}\right) \alpha_{m}
$$

where

$$
\begin{aligned}
& R_{1}=-\frac{1}{2} f_{1}=-\frac{1}{2}\left(\frac{N_{2}}{N_{1}}-\frac{D_{2}}{D_{1}}+2 \frac{\bar{\zeta}_{3}}{\bar{\zeta}_{2}}-1\right), \\
& R_{0}=-\frac{1}{2} \frac{\alpha_{0}}{\alpha_{m}} f_{0}=-\frac{1}{2}\left[1-\psi^{\prime}\left(\gamma_{s}+1 / 2\right) \gamma_{s}\right] f_{0},
\end{aligned}
$$

and $f_{0}=\left(3-\tau^{2}\right) /(1-\tau)(3-\tau)$. The functions $N_{1}, D_{1}$ are defined in Eq. (12) and

$$
\begin{aligned}
N_{2} & =3(1-\tau)^{2}\left[\bar{\zeta}_{2}-2 \bar{\zeta}_{3}+\bar{\zeta}_{4}\right]+\tau(2-\tau)-\bar{\zeta}_{2} \\
D_{2} & =2\left(3 \bar{\zeta}_{4}-\bar{\zeta}_{3}-2 \bar{\zeta}_{5}\right)
\end{aligned}
$$

Furthermore, Eq. (15) has been written using the relation for the isotope fefficient of $T_{c}$ in the presence of magnetic impurities:2 20

$$
\alpha_{m}=\frac{\alpha_{0}}{1-\psi^{\prime}\left(\gamma_{s}+1 / 2\right) \gamma_{s}},
$$

where $\alpha_{0}$ is the isotope coefficient of $T_{c 0}$, the critical temperature in the absence of magnetic impurities. $\psi^{\prime}$ is the derivative of the psi function.

Eq. (13) shows that the isotope coefficient of $\delta$ is proportional to the isotope coefficient of $T_{c}$. This relation is valid near $T_{c}$ (where $\Delta$ is small) and for impurity concentrations such that the condition $\Delta T_{c} / T_{c} \ll 1$ is satisfied.

An important feature of the result (13) is that the IC of $\delta$ is a universal function $\beta\left(T / T_{c}\right)$. Indeed, Eq. (13) can be calculated once $T / T_{c}, T_{c 0}, \alpha_{0}$ and $\gamma_{s}$ are given. The three first quantities are obtained from experiment. The last parameter $\gamma_{s}$ also determines the depression of $T_{c}$ induced by magnetic impurities and can be calculated from the Abrikosov-Gor'kov equation 18 :

$$
\ln \left(\frac{T_{c 0}}{T_{c}}\right)=\psi\left(\frac{1}{2}+\gamma_{s}\right)-\psi\left(\frac{1}{2}\right)
$$

From Eqs. (13), 16) and (17) we thus conclude that the knowledge of the measurable quantities $\alpha_{0}, T_{c 0}$ and $T / T_{c}$ completely determines the IC $\tilde{\beta}_{m}$.

If besides the magnetic impurity channel there is also a contribution from the non-adiabatic channel as presented in the last section, the total IC takes the form $\beta_{t o t}=\beta_{p h}+\beta_{m}+\beta_{n a}$. In addition, according to Ref. 2, one has to replace $\alpha_{0}$ in Eq. (16) by $\alpha_{n a}+\alpha_{p h}$ ( $\alpha_{n a}$ is given in Eq. (位) and $\alpha_{p h}$ is the usual phonon contribution to the IC of $T_{c}$ ). The non-adiabatic and magneticimpurity contributions to the IC are thus non-additive near $T_{c}$ (because of the presence of $\alpha_{n a}$ in $\beta_{m}$ and the presence of $\Gamma_{2}$ in $\beta_{p h}$; as shown in Sec. II A 2, $\beta_{n a}$ is unaffected by magnetic impurities). One notes further, that if the IC is induced by magnetic impurities only, then its value is always negative, whereas the situation might be different in the presence of a non-adiabatic contribution.

The factor $\beta_{0}$ in Eq. (13) which is the IC of $\delta$ in the absence of magnetic impurities contains a trivial temperature dependence due to the factor $\varphi\left(T / T_{c}\right) \sim 1-\left(T / T_{c}\right)^{4}$ and the isotopic dependence of $T_{c}$. However, the presence of magnetic impurities leads to an additional, novel temperature dependence of $\tilde{\beta}_{m}$, beyond the BCS one. This temperature dependence is shown in Fig. 1 for fixed values of $T_{c}$ (i.e. of the magnetic impurity concentration). The solid and dashed lines describe the case where only magnetic impurities are added to the system (no nonadiabatic contribution). The situation corresponds to Zn-doped YBCO (YBCZnO). It has been shown with use of NMR technique that $\mathrm{Zn}$-substitution (for $\mathrm{Cu}$ ) occurs in the $\mathrm{CuO}_{2}$ plane and leads to the appearence of localized magnetic moments (see Ref. 21 and below). The two other curves (dotted and dash-dotted lines) are obtained when, in addition, a non-adiabatic channel is present. They are discussed below. Fig. 1 shows that the absolute value of the IC increases as one approaches $T_{c}$. This temperature dependency is significant since in certain cases the value of $\beta$ triples when $\tau=0.75$ increases to $\tau=0.95$. Note also, that the solid and dashed lines describe conventional superconductors as well, since only $\alpha_{0}$ has to be modified (in YBCZnO $\alpha_{0}=\alpha_{p h}=0.025$ which is much smaller than typical values for conventional superconductors).

Fig. 2 shows the dependency of the IC $\tilde{\beta}_{m}$ on $T_{c}$ (i.e. on the concentration $n_{M}$ ) for fixed values of the temperature. It appears that an increase of magnetic impurity concentration leads to an increase of $\left|\tilde{\beta}_{m}\right|$. Moreover, the change of $\tilde{\beta}_{m}\left(T_{c}\right)$ is stronger as $T$ approaches $T_{c}$. It turns out that the qualitative behaviour of $\tilde{\beta}_{m}\left(T_{c}\right)$ is similar to $\alpha_{m}\left(T_{c}\right)$ [Eq. (16) with $\alpha_{0}=\alpha_{p h}$ ] but with opposite sign (see also Fig. 1 of Ref. 2). As for Fig. 1, the parameter $\alpha_{0}$ corresponds to the value for $\mathrm{YBCZnO}$, but the qualitative picture holds for conventional superconductors as well.

The calculation of the IC near $T_{c}$ presented above suggests an experiment to determine the contribution of magnetic impurities to the IC. For conventional superconductors where the magnetic moments are introduced into the superconductor as impurities one can substract the BCS isotope coefficient discussed at the end of last section by measuring its temperature dependency. For high-temperature superconductors the same procedure can in principle be applied. Nevertheless, one has to be more carefull since magnetic impurities are intrinsically present in some of these materials as shown in Ref. 22. Indeed, there are evidences that already optimally doped YBCO contains magnetic impurities. Upon depletion of oxygen one further adds magnetic impurities. One can thus not avoid the presence of these localized magnetic moments. As a consequence, it is more difficult to extract the BCS component for these materials. The procedure 
in high- $T_{c}$ superconductors would thus consist in measuring the (temperature dependent) isotope coefficient of $\delta$ at optimal doping (which contains the smallest concentration of intrinsic magnetic moments) and substract this component from the measurements at a given doping. This applies, e.g., to oxygen-depleted, Pr or Zn-doped YBCO (see also Refs. 21,22).

Note that our analysis in this section is based on the theory in Ref. 18 (see Eq. (17)). The core of this theory is the introduction of the pair-breaking effect. This effect requires singlet pairing and is applicable to both $s$ and $d$-wave symmetries of the order parameter. There is, of course, a quantitative difference between these two cases, but the isotope effect for the penetration depth should be observed, regardless of the symmetry. In this paper, in analogy with Ref. 2, we make specific calculations for the $s$-wave scenario. Therefore, these calculations are valid, even quantitatively for conventional superconductors, as well as for some oxides (e.g. Nd-based cuprates, Ba-PbBi-O). As for the YBCO compound, our calculations of the isotope shift for $T_{c}$ caused also by magnetic impurities, revealed excellent agreement with the data (see Ref. 2, Fig. 1a) which is interesting from the point of view of analyzing the complex problem of the symmetry. In this paper, we employ a similar approach. It is essential, however, to note that, as mentioned above, the qualitative picture is similar for both scenarios ( $s$ or $d$-wave).

The results presented in Figs. 1 and 2 were calculated on the basis of the parameters for $\mathrm{Zn}$-doped $\mathrm{YBCO}$ (YBCZnO) and consider solely the influence of magnetic impurities on $\beta$. The situation is more complicated if, in addition, non-adiabaticity is present in the system. In this case $\tilde{\beta}_{m}$ is given by Eqs. (13) to (17) and $\alpha_{0}$ by Eq. (4). To stress the influence of non-adiabaticity on $\tilde{\beta}_{m}$ through $\alpha_{0}$ we write $\tilde{\beta}_{m+n a}$ in the following. We have calculated the IC of the penetration depth near $T_{c}$ for Prdoped YBCO (YPrBCO) using the parameters derived in Ref. 2. The results are shown in Figs. 1 (dotted and dotdashed lines) and 3 for the temperature and magnetic impurity concentration dependence, respectively.

Several comments can be made by analyzing these figures. Fig. 1 shows that there is no qualitative change in $\beta(T)$ (at fixed $T_{c}$ ) when adding the non-adiabatic contribution. Non-adiabaticity merely induces a stronger temperature dependency. This may, however, be specific to the materials studied here. On the other hand, contrary to the case of magnetic impurities alone (Fig. 2), there is a qualitative difference (besides the opposite signs) between the IC of $T_{c}$ and $\delta$ taken as a function of $T_{c}$ and in the presence of non-adiabaticity. To understand this, one has to come back to Eqs. $(13)-(15)$. Let us first rewrite Eq. (13) in the form

$$
\tilde{\beta}_{m}=\beta_{m}-\beta_{0}=-\frac{1}{2}\left(f_{1} \alpha_{m}-f_{0} \alpha_{0}\right)
$$

where $f_{0}$ and $f_{1}$ are defined in Eqs. (14), (15). It turns out that $f_{1}\left(T / T_{c}\right)$ and $f_{0}\left(T / T_{c}\right)$ are never more than sev- eral percents apart from each other for given $T$ and $T_{c}$. The shape of $\tilde{\beta}_{m+n a}$ in the presence of non-adiabaticity and magnetic impurities is thus mainly determined by the difference $\alpha_{m}-\alpha_{0}$ [where $\alpha_{m}$ is given by Eq. (16) and $\alpha_{0}=\alpha_{p h}+\alpha_{n a}$ by Eq. (4)]. Using a polynomial expansion of $T_{c}(n)$ (as done in Ref. 2) one can show that $\alpha_{0}$ saturates with decreasing $T_{c}\left[\alpha_{n a}\left(T_{c} \rightarrow 0\right) \rightarrow \gamma\right.$, with $\gamma$ defined in Eq. (4)], whereas $\alpha_{m}$ increases as $n_{M}^{2}$ in the same limit (see Ref. 2). Thus, at high impurity doping, the behaviour of $\tilde{\beta}_{m+n a}$ is dominated by the denominator of Eq. (16), that is by the magnetic impurities. In the opposite low doping regime, $\tilde{\beta}_{m+n a}$ is dominated by the non-adiabatic part in $\alpha_{m}$ and $\alpha_{0}$. Accordingly, $\tilde{\beta}_{m+n a}$ behaves similarly near optimal $T_{c}$ and near the $60 \mathrm{~K}$ plateau (in YBCO), where $\partial T_{c} / \partial n$ is small. Thus, as can be seen on Fig. $3 \mathrm{~b}, \tilde{\beta}_{m+n a}$ behaves approximately as $-\alpha_{n a}$ in these regions but is smaller by an amount approximately equivalent to $\alpha_{0}$. As one gets away from the plateaus, the magnetic impurity part of $\alpha_{m}-\alpha_{0}$ becomes dominant, leading to a departure from the behaviour of $\alpha_{n a}$. The fact that $\tilde{\beta}_{m+n a}$ is mainly determined by $\alpha_{m}-\alpha_{0}$ (instead of $\alpha_{m}$ for the IC of $T_{c}$ ) explains why the change of curvature of $\tilde{\beta}_{m+n a}$ (see Fig. $3 \mathrm{~b}$ ) takes place at higher $T_{c}$ 's (lower magnetic impurity concentrations) than the IC of $T_{c}, \alpha_{m}$ (see also Fig. 2a of Ref. 2). The change of curvature should be measurable since the precision of measurements increases with de-

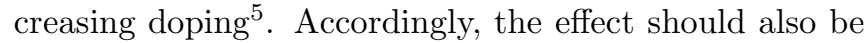
observable for oxygen-depleted YBCO for $T_{c}$ near its optimal value and below but near the $60 \mathrm{~K}$ plateau.

We have not calculated the penetration depth IC for oxygen-depleted YBCO near $T_{c}$ because the dependencies $\Gamma_{s}(x)$ (the spin-flip scattering amplitude as a function of oxygen doping) and $n(x)$ are not well known for this material. This was not a major problem for the calculation of the IC $\alpha_{m}$ of $T_{c}$, because $\alpha_{m}$ is mainly determined by the non-adiabatic channel in the regime where the experiments were performed and is affected by magnetic impurities in the higher doping (i.e. lower $T_{c}$, away from the plateaus) regime (see Eq. (16) and Ref. 22). However, it might be of importance for the calculation of the IC of $\delta$ because of the more complicated structure of the relation $\beta_{m}\left(\Gamma_{s}\right)$. This case is discussed in more detail in Ref. 3.

Finally, one should note that there is no apparent reason for the IC of $\delta$ to be restricted to values below 0.5 . In fact, the calculations show that at high enough dopant concentration, the effect should be very large (this is also true at $T=0$ as shown in the next section).

\section{B. Magnetic impurity contribution to $\beta$ at $T=0$}

Since we want to determine solely the contribution of non-adiabaticity and magnetic impurities to the IC, one is interested in eliminating the presence of the BCS contribution caused by the factor $\varphi\left(T / T_{c}\right)$ and the usual 
isotopic dependence of $T_{c}$. Besides the procedure presented in the last section there is another way to avoid $\beta_{B C S}$. Indeed, the BCS part becomes negligibly small $\left(\varphi \sim 1-\left(T / T_{c}\right)^{4}\right)$ as one lowers the temperature. At

$$
\tilde{K}(0,0)=-\frac{1+\bar{\Gamma} \bar{\eta}^{-3}}{\bar{\eta}}\left[\frac{\pi}{2}-\frac{f(\bar{\eta})}{R(\bar{\eta})}\right]+\bar{\Gamma} \bar{\eta}^{-3}\left[\frac{2}{3} \bar{\eta}-\frac{\pi}{4} \bar{\eta}+1\right]
$$

for $\bar{\Gamma}, \bar{\eta}<1$ (with $f(\bar{\eta})=\operatorname{arcos} \bar{\eta})$ or $\bar{\Gamma}<1, \bar{\eta}>1($ with $f(\bar{\eta})=\operatorname{arcosh} \bar{\eta})$ and

$$
\begin{aligned}
\tilde{K}(0,0)= & -\frac{1+\bar{\Gamma} \bar{\eta}^{-3}}{\bar{\eta}}\left\{\frac{\pi}{2}-2 \frac{\bar{\Gamma}-1}{R(\bar{\Gamma})}-R^{-1}(\bar{\eta})(\operatorname{arcosh} \bar{\eta}-2 \operatorname{artanh} \mathcal{R})\right\} \\
& +\bar{\eta}^{-3}\left\{\left(\frac{2}{3} \bar{\eta}^{2}+1\right)(\bar{\Gamma}-R(\bar{\Gamma}))-\frac{1}{2} \eta R(\bar{\Gamma})\left(\frac{2}{3} \eta-1\right)-\bar{\eta} \bar{\Gamma}\left(\frac{\pi}{4}-\frac{\bar{\Gamma}-1}{R(\bar{\Gamma})}\right)\right\}
\end{aligned}
$$

for $\bar{\Gamma}, \bar{\eta}>1$. We have introduced the notation $\bar{\Gamma}=$ $\Gamma_{s} / \Delta, \bar{\eta}=\eta \bar{\Gamma}=\Gamma_{2} / \Delta, \eta=\Gamma_{2} / \Gamma_{s}, R(x)=\sqrt{\left|1-x^{2}\right|}$ with $x=\bar{\Gamma}, \bar{\eta}$ and $\mathcal{R}=[(\bar{\Gamma}-1)(\bar{\eta}-1) /(\bar{\Gamma}+1)(\bar{\eta}+1)]^{1 / 2}$. $\Delta \equiv \Delta\left(T=0, \Gamma_{s}\right)$ is the order parameter in the presence of magnetic impurities. Eqs. (19), (20) are valid when $\Gamma_{s} \ll \Gamma_{2}$. These two scattering amplitudes, $\Gamma_{2}$ and $\Gamma_{s}$ $\left(\Gamma_{s}=\Gamma_{1}-\Gamma_{2}\right)$, defined by Abrikosov and Gor'kov 18 , describe the direct and exchange scattering, respectively. One can calculate the magnetic impurity contribution to the IC at $T=0$ from Eq. (11) in a straightforward way using Eqs. (19) and (20). The result can be written as

$$
\beta_{m}(T=0)=-\frac{\alpha_{\Delta}}{2} \frac{K_{1}+K_{2}}{\tilde{K}(0,0)}
$$

where $\alpha_{\Delta}$ is defined below, $\tilde{K}(0,0)$ is given by Eqs. (19), (20) and

$$
\begin{aligned}
& K_{1}=-\frac{1+3 \bar{\Gamma} \bar{\eta}^{-3}}{\bar{\eta}}\left(\frac{\pi}{2}-\frac{f(\bar{\eta})}{R(\bar{\eta})}\right) \pm \frac{1+\bar{\Gamma} \bar{\eta}^{-3}}{R(\bar{\eta})^{2}}\left(\frac{\bar{\eta}}{R(\bar{\eta})} f(\bar{\eta})-1\right) \\
& K_{2}=\bar{\Gamma} \bar{\eta}^{-3}\left(2-\frac{\pi}{4} \bar{\eta}\right)
\end{aligned}
$$

for $\bar{\Gamma}, \bar{\eta}<1$ (upper sign, $f(\bar{\eta})=\operatorname{arcos} \bar{\eta})$ or $\bar{\Gamma}<1, \bar{\eta}>1$ (lower sign, $f(\bar{\eta})=\operatorname{arcosh} \bar{\eta})$ and

$$
\begin{aligned}
K_{1}= & -\frac{1+3 \bar{\Gamma} \bar{\eta}^{-3}}{\bar{\eta}}\left\{\frac{\pi}{2}-2 \frac{\bar{\Gamma}-1}{R(\bar{\Gamma})}-\frac{1}{R(\bar{\eta})}(\operatorname{arcosh} \bar{\eta}-2 \operatorname{artanh} \mathcal{R})\right\}-\frac{1+\bar{\Gamma} \bar{\eta}^{-3}}{\bar{\eta}}\left\{2 \frac{\bar{\Gamma}(\bar{\Gamma}-1)}{R(\bar{\Gamma})^{3}}\right. \\
& \left.-\frac{1}{R(\bar{\eta})}\left[\frac{\bar{\eta}}{R(\bar{\eta})^{2}}(\operatorname{arcosh} \bar{\eta}-2 \operatorname{artanh} \mathcal{R})+\frac{\mathcal{R}}{1-\mathcal{R}^{2}}\left(\frac{\bar{\Gamma}^{2}}{R(\bar{\Gamma})^{2}}+\frac{\bar{\eta}^{2}}{R(\bar{\eta})^{2}}\right)-\frac{\bar{\eta}}{R(\bar{\eta})}\right]\right\} \\
K_{2}= & \frac{3}{\bar{\eta}^{3}}\left(\frac{2}{3} \bar{\eta}^{2}+1\right)[\bar{\Gamma}-R(\bar{\Gamma})]-\frac{1}{2} \eta R(\bar{\Gamma})\left(\frac{2}{3} \eta^{2}-1\right)-\bar{\eta} \bar{\Gamma}\left(\frac{\pi}{4}-\frac{\bar{\Gamma}-1}{R(\bar{\Gamma})}\right) \\
& +\frac{1}{\bar{\eta}^{3}}\left\{\left[\frac{\bar{\Gamma}}{R(\bar{\Gamma})}\left(\frac{2}{3} \bar{\eta}^{2}+1\right)-\frac{4}{3} \bar{\eta}^{2}\right][\bar{\Gamma}-R(\bar{\Gamma})]\right. \\
& \left.+\frac{1}{2} \eta \frac{\bar{\Gamma}^{2}}{R(\bar{\Gamma})}\left(\frac{2}{3} \eta^{2}-1\right)+2 \bar{\eta} \bar{\Gamma}\left[\frac{\pi}{4}-\frac{\bar{\Gamma}-1}{R(\bar{\Gamma})}\left(1+\frac{\bar{\Gamma}}{2 R(\bar{\Gamma})^{2}}\right)\right]\right\}
\end{aligned}
$$

for $\bar{\Gamma}, \bar{\eta}>1$ and $\Delta T_{c} / T_{c}(\bar{\Gamma}-1) \ll 1$. The last condition expresses the fact that the calculation is not valid in the immediate vicinity of $\bar{\Gamma}=1$. Eq. (21) contains $\alpha_{\Delta}$ which is the IC of the order parameter $\Delta$. In strong- coupling systems, $\alpha_{\Delta}$ has to be calculated numerically using Eliashberg's equations. In the following we calculate the IC in the framework of the BCS model where $\alpha_{\Delta}$ can be calculated analytically. Indeed, from the relations

$$
\ln \left(\frac{\Delta}{\Delta_{0}}\right)=\left\{\begin{array}{ll}
-\frac{\pi}{4} \bar{\Gamma} & : \bar{\Gamma} \leq 1 \\
-\ln [\bar{\Gamma}+R(\bar{\Gamma})]+\frac{R(\bar{\Gamma})}{2 \bar{\Gamma}}-\frac{\bar{\Gamma}}{2} \arctan R(\bar{\Gamma})^{-1} & : \bar{\Gamma}>1
\end{array},\right.
$$

derived by Abrikosov and Gor'kov $\left[\Delta_{0}=\Delta\left(T=0, \Gamma_{s}=0\right)\right.$ is the order parameter in the absence of magnetic impurities] one obtains 


$$
\alpha_{\Delta}=\alpha_{\Delta_{0}} \begin{cases}\left(1-\frac{\pi}{4} \bar{\Gamma}\right)^{-1} & : \bar{\Gamma} \leq 1 \\ {\left[1-\frac{\bar{\Gamma}}{2} \arctan R(\bar{\Gamma})^{-1}-\frac{R(\bar{\Gamma})}{2 \bar{\Gamma}}\right]^{-1}} & : \quad \bar{\Gamma}>1\end{cases}
$$

In the BCS approximation one further has $\alpha_{\Delta_{0}}=\alpha_{0}$, where the last quantity was defined before as the IC of $T_{c 0}$, that is, in the absence of magnetic impurities. As for the calculation near $T_{c}$, the isotope coefficient $\beta_{m}$ at $T=0$ is independent of any free parameter. Indeed, $\Gamma_{s}$ is determined by the Abrikosov-Gor'kov equation relating $T_{c}$ and $T_{c 0}$, whereas $\Gamma_{2}$ (the direct scattering amplitude) is determined via normal state properties of the material. Thus the relation $\beta_{m}\left(T_{c}\right)$ is also universal at $T=0$.

Since the penetration depth $\delta$ does not depend explicitly on $T_{c}$ at $T=0$ [see Eqs. (19) and (20)], $\beta_{m}(T=0)$ is independent of the isotope coefficient $\alpha$. Consequently, the contributions of magnetic impurities and the nonadiabatic channel to the IC of $\delta$ are simply additive at $T=0$. This has to be seen in contrast to the calculation of the last section performed near $T_{c}$. In the following we neglect the additive non-adiabatic additive contribution and show only $\beta_{m}$.

The numerical calculation of $\beta_{m}$ from Eqs. (22)-(25) is shown in Figs. $4 \mathrm{a}$ and $4 \mathrm{~b}$ as a function of $T_{c}$ (that is of the magnetic impurity concentration) for two values of the ratio $\eta=\Gamma_{2} / \Gamma_{s}(>1)$. Fig. 4a displays the case of a conventional superconductor and Fig. $4 \mathrm{~b}$ was obtained with parameters corresponding to the situation of hightemperature superconductors. The result shows that in analogy with the IC of $T_{c}$ in the presence of magnetic impurities and with the calculations of the last section, $\left|\beta_{m}(T=0)\right|$ increases with increasing magnetic impurity concentration. However, the two coefficients $\alpha$ and $\beta$ have opposite signs.

There are two main differences between the situations of conventional and high-temperature superconductors. On the one hand, the isotope coefficient $\beta_{m}$ at $T=0 \mathrm{~K}$ of high- $T_{c}$ superconductors remains small over a relatively large concentration range of magnetic impurities. The effect becomes sizeable in the gapless regime. The increase of $\left|\beta_{m}(T=0)\right|$ is much larger at low doping for conventional superconductors. As a consequence, on the other hand, the effect of the parameter $\eta$ is sizeable only in the case of conventional superconductors and in the low impurity concentration regime. The calculations suggest also that for both types of superconductors the largest effect is observed at low $T_{c}$ where the superconductor is in the gapless state $(\bar{\Gamma}, \bar{\eta}>1)$. The values of the IC are then of the same order as those of the IC of $T_{c}$. It would be interesting to perform experiments both on conventional and high-temperature superconductors. As discussed before, Zn- or Pr-doped YBCO are interesting candidates since the IC of $T_{c}$ as a function of doping (which is necessary in order to cadclate $\left.\beta_{t o t}=\beta_{p h}+\beta_{m}+\beta_{n a}\right)$ is known in both cases. 2 .

\section{ISOTOPE EFFECT OF $\delta$ IN PROXIMITY SYSTEMS}

In this last section we consider another physical situation, a proximity system, in which a factor not related to lattice dynamics, induces an IE of the penetration depth. We have shown in Ref. 2 that the presence of a normal film on a superconductor induces a change in the IC of $T_{c}$. Here we show that it also induces an isotopic shift of $\delta$. We study a proximity system of the type $S-N$, where $S$ is a weak-coupling superconductor and $N$ is a metal or a semiconductor (e.g. $\mathrm{Pb}-\mathrm{Ag}$ ). Let us note $T_{c 0}$ the value of the critical temperature for an isolated $S$ film. As is known, the proximity effect leads to a critical temperature $T_{c}$ that differs from $T_{c 0}$ (see, e.g., Refs. 2,23). The proximity effect also affects the shielding of a weak magnetic field. The most dramatic effect of the normal layer on the penetration depth is seen in the low temperature regime $\left(T / T_{c} \leq 0.3\right)$. Although the penetration depth of a pure conventional superconductor is only weakly temperature dependent in this regime $\left(\delta \sim \sqrt{1-T^{4} / T_{c}^{4}}\right)$ the presence of the normal layer induces a temperature dependence through the proximity effect.

Consider a proximity system $S-N$ and assume that $\delta<L_{N} \ll \xi_{N}$ (which is certainly satisfied in the lowtemperature regime since $\xi_{N}=\hbar v_{F} / 2 \pi T$; see, e.g., Ref. 24), where $L_{N}$ and $\xi_{N}$ are the thickness and the coherence length of the normal film, respectively (cf. Ref. 2 and references therein). In this case, the penetration depth $\delta$ has been calculated by one of the author 24 :

$$
\delta^{-3}=a_{N} \Phi \quad
$$

where $a_{N}$ is a constant depending only on the material properties of the normal film (it is independent of the ionic mass) and

$\Phi=\pi T \sum_{n \geq 0} \frac{1}{x_{n}^{2} p_{n}^{2}+1}, \quad p_{n}=1+\varepsilon t \sqrt{x_{n}^{2}+1}$.

$x_{n}=\omega_{n} / \epsilon_{S}(T)$ with $\omega_{n}=(2 n+1) \pi T$ (the Mastubara frequencies) and $\epsilon_{S}(T)$ is the superconducting energy gap of $S$. In the weak-coupling limit considered here, $\epsilon_{S}(0)=\varepsilon \pi T_{c 0}$ with $\varepsilon \simeq 0.56$. The dimensionless parameter $t=\ell / S_{0}$ with $\ell=L_{N} / L_{0}$ and $S_{0}=\Gamma_{0} / \pi T_{c 0}$ $\left(\Gamma_{0} \sim 1 / L_{0}\right.$ is the McMillan parameter 25$)$. $L_{N}$ and $L_{0}$ are the thickness of the normal film and some arbitrary thickness, respectively (in the following we take $L_{0}=L_{S}$, the thickness of the superconducting film).

Since $\delta$ depends non-linearly on $T_{c 0}$ (through the proximity parameter $t$ ), the penetration depth will display an 
isotope shift due to the proximity effect. From Eq. 26 one can calculate the isotope coefficient $\beta_{\text {prox }}$ [as defined by Eq. (1)] of the penetration depth due to the proximity effect:

$$
\beta_{\text {prox }}=-\frac{2 \alpha_{0}}{3 \Phi} \sum_{n>0} \frac{x_{n}^{2} p_{n}^{2}}{x_{n}^{2} p_{n}^{2}+1}\left(1-\frac{\varepsilon t}{p_{n} \sqrt{x_{n}^{2}+1}}\right),
$$

where $\alpha_{0}$ is the IC of $T_{c 0}$ for the superconducting film $\mathrm{S}$ alone.

This result has several interesting properties. First, it turns out that the isotope coefficients $\beta_{\text {prox }}$ and $\alpha_{0}$ have generally opposite signs (see below). Secondly, the IC depends on the proximity parameter $t$, that is on the thickness ratio $\ell=L_{N} / L_{S}$ of the normal and superconducting films and on the MacMillan tunneling parameter $\Gamma_{0}$.

The dependency on the ratio $\ell$ has already been mentioned in connection with the IE of $T_{c}^{2}$, 3 . Fig. 5 shows the isotope coefficient $\beta_{\text {prox }}$ as a function of the parameter $t$ for fixed temperatures. $\left|\beta_{\text {prox }}\right|$ decreases with increasing $t$. There are two ways to change the value of $t \sim \ell / S_{0}$. One of them is an increase of the parameter $\ell=L_{N} / L_{S}$ (e.g. an increase of the thickness of the normal film $L_{N}$ ). Note that there is a lower bound $\ell_{\min }$ to the value of $\ell$, since it was assumed that $\delta<L_{N}$ to obtain Eq. (26) 24 . The IC $\left|\beta_{\text {prox }}\right|$ decreases with increasing thickness ratio (Fig. 5 then corresponds to the values $S_{0}=0.2$ for $\left.\left.\ell \in] \ell_{\text {min }}, 1\right]\right)$. However, the higher the temperature the smaller the decrease of $\beta_{\text {prox }}$ which then rapidly reaches saturation with increasing thickness.

Instead of changing the thickness ratio $\ell$ one can vary the parameter $\Gamma_{0}$. One possibility is to use different films, another is to modify the quality of the $S-N$ interface. Fig. 5 then implies that the $\left|\beta_{\text {prox }}\right|$ decreases with decreasing tunneling parameter $\Gamma_{0}$. It would be interesting to perform an experiment where the isotope effect of $T_{c}$ and $\delta$ are determined for a proximity system with different values of the thickness ratio $\ell$.

Note that the result (28) has another interesting feature ; one can see that the isotopic shift of the penetration depth is temperature dependent. Fig. 6 shows the temperature dependence of $\beta_{\text {prox }}$ for two sets of parameters. The two upper curves are obtained for $S_{0}=0.2$ and two thickness ratios $\ell=0.5,1$; the two lower curves are for $S_{0}=1$ and $\ell=0.5,1$. From Fig. 6 one concludes that $\left|\beta_{\text {prox }}\right|$ increases with increasing temperature. Furthermore, a weak tunneling between the superconducting and the normal film (smaller $S_{0}$ ) induces a strong temperature dependence. Finally, the temperature range over which the IC remains unchanged is broader for a larger than a smaller tunneling parameter.

Therefore, the presence of a normal layer on a superconductor induces an isotope shift of the penetration depth. Moreover, in a proximity system the IC depends on the thickness ratio and the temperature. The values of the isotope coefficient are such that they are measurable in conventional and high-temperature superconductors.

\section{CONCLUSION}

We have shown that the value of such a fundamental parameter as the penetration depth $\delta$ depends on isotopic substitution. This unconventional dependency is caused by number of factors not related to the pairing mechanism.

At first, we focused on the non-adiabatic IE. This effect, manifested in the dependence of the charge-carrier concentratipg on the ionic masses for doped systems such as cuprates 2 , leads to a noticeable isotopic shift of the penetration depth $\delta$. For this case, we established a general relation between the isotope coefficient of $T_{c}$ and of $\delta$ for London superconductors (see Eq. (10)). The isotope coefficient of $\delta$ is a constant independent of doping or temperature; we determined its value for oxygen depleted and $\mathrm{Pr}$ substituted $\mathrm{YBa}_{2} \mathrm{Cu}_{3} \mathrm{O}_{7-\delta}$ as well as for $\mathrm{La}_{2-x} \mathrm{Sr}_{x} \mathrm{CuO}_{4}$ Note that similar effects are expected in manganites 10 where the charge-transfer processes also involve the non-adiabatic oxygen ions.

The presence of magnetic impurities also leads to a non-trivial isotope effect for $\delta$. We studied this effect for temperatures near $T_{c}$ and at $T=0$. It turns out that the coefficient obeys a universal law; it is determined by the experimental values of $T_{c}$ in the absence and presence of magnetic impurities [Eqs. (13) and (21)] as well as the isotope coefficient of $T_{c}$ in the absence of magnetic impurities. The combined effect of magnetic impurities and non-adiabaticity was also discussed. Finally, we showed that the penetration depth for proximity systems also display an isotopic shift. It is interesting that in the case of a proximity system or in the presence of magnetic impurities the IC for $\delta$ is temperature dependent.

All these effects should be measurable in conventional and in high-temperature superconductors and several experiments were proposed. In the case of high- $T_{c}$ materials it would be of interest to study oxygen depleted $\mathrm{YBa}_{2} \mathrm{Cu}_{3} \mathrm{O}_{7-\delta}$ as well as the Pr- and Zn-substituted systems, since the IE of $T_{c}$ has been successfully described in these materials (see Refs. 113). The case of Zn-substituted $\mathrm{YBa}_{2} \mathrm{Cu}_{3} \mathrm{O}_{6+x}$ is here of special interest, since experiments show that the depression of $T_{c}$ is mainly due to the change in magnetic impurity concentration and our theory is free of any adjustable parameter in this case.

Note, finally, that not only $T_{c}$ and $\delta$ exhibit the unconventional isotope effects studied here and in Refs. 113, but any quantity that depends on the charge carrier density of the superconducting condensate or/and on $T_{c}$ (e.g. the specific heat).

A.B. is grateful to the Swiss National Science Foundation and the Naval Research Laboratory for financial support. The work of V.Z.K. is supported by the U.S. Office of Naval Research under contract No. N00014-96-F0006. 
${ }^{1}$ V.Z. Kresin, and S.A. Wolf, Phys. Rev. B 49, 3652 (1994); and in Anharmonic Properties of High-T $T_{c}$ Cuprates, p. 18, D. Mihailovic, G. Ruani, E. Kaldis, K.A. Müller, Eds., World Scientific (1995).

${ }^{2}$ V.Z. Kresin, A. Bill, S.A. Wolf, and Yu.N. Ovchinnikov, Phys. Rev. B 56, 107 (1997).

${ }^{3}$ A. Bill, V.Z. Kresin, and S.A. Wolf, Z. Phys. B, in press; A. Bill, and V.Z. Kresin, Proceedings of the International Symposium on Electrons and Vibrations in Solids and Finite Systems, Berlin, August 1996, U. Scherz and H.J. Schulz eds., Z. Phys. Chem. 201, 271 (1997).

${ }^{4}$ J.P. Franck, J. Jung, M.A-K. Mohamed, S. Gygax, and G.I. Sproule, Phys. Rev. B 44, 5318 (1991).

${ }^{5}$ G. Soerensen, and S. Gygax , Phys. Rev. B 51, 11848 (1995).

${ }^{6}$ D. Zech, K. Conder, H. Keller, E. Kaldis, and K.A. Müller, Physica B 219\&220, 136 (1996).

${ }^{7}$ H.J. Bornemann and D.E. Morris, Phys. Rev. B 44, 5322 (1991).

${ }^{8}$ G.-M. Zhao and D.E. Morris, Phys. Rev. B 51, 16487 (1995).

${ }^{9}$ G.-M. Zhao, K.K. Singh, A.P.B. Sinha, and D.E. Morris, Phys. Rev. B 52, 6840 (1995); G.-M. Zhao, M.B. Hunt, H. Keller, and K.A. Müller, Nature 385, 236 (1997); G. Deutscher, Proceedings of the International School on The Gap Symmetry and Fluctuations in High-T $T_{c}$ Superconductors, Cargèse (1997).

${ }^{10}$ V.Z. Kresin, and S.A. Wolf, Phil. Mag. 76, 241 (1997).

${ }^{11}$ J. Mustre de Leon, S.D. Conradson, I. Batistic, and A.R. Bishop, Phys. Rev. Lett. 65, 1675 (1990); L.V. Gasparov, V.D. Kulakovskii, V.B. Timofeev, and E.Ya. Sherman, J. Supercond. 8, 27 (1995); G. Ruani, P. Guptasarma, and C. Taliani, Solid State Comm. 96, 653 (1995); A. Jezowski, J. Klamut, and B. Dabrowski, Phys. Rev. B 52, 7030 (1995).

12 R.P. Sharma, T. Venkatesan, Z.H. Zhang, J.R. Liu, R. Chu, and W.K. Chu, Phys. Rev. Lett. 77, 4624 (1997); D. Haskel, E.A. Stern, D.G. Hinks, A.W. Mitchell, and J.D. Joergensen, Phys. Rev. B 56, 521 (1997).

${ }^{13}$ K.H. Johnston, D.P. Clougherty, and M.E. McHenry, in Novel Superconductivity, p. 563, S. Wolf and V.Z. Kresin, Eds., Plenum, NY (1986).

14 T.F. O'Malley, Phys. Rev. 162, 98 (1967).

15 E.A. Lynton, Superconductivity, p. 37, Methuen Eds., 1969.

${ }^{16}$ M.K. Crawford, M.N. Kunchur, W.E. Farneth, E.M. Mc-
Carron III, and S.J. Poon, Phys. Rev. 41, 282 (1990)

17 M.I. Salkola, V.J. Emery, and S.A. Kivelson, Phys. Rev. Lett. 77, 155 (1996); B. Dabrowski, Z. Wang, K. Rogacki, J.D. Jorgensen, R.L. Hitterman, J.L. Wagner, B.A. Hunter, P.G. Radaelli, and D.G. Hinks, Phys. Rev. Lett. 76, 1348 (1996); Physica C 217, 455 (1993); N. Yamada and M. Ido, Physica C 203, 240 (1992); P.G. Radaelli, D.G. Hinks, A.W. Mitchell, B.A. Hunter, J.L. Wagner, B. Dabrowski, K.G. Vandervoort, H.K. Viswanathan, and J.D. Jorgensen, Phys. Rev. B 49, 4163 (1994); B. Büchner, M. Breuer, A. Freimuth, and A.P. Kampf, Phys. Rev. Lett. 73, 1841 (1994); M.R. Norman, G.J. McMullan, D.L. Novikov, and A.J. Freeman, Phys. Rev. B 48, 9935 (1993).

18 A. Abrikosov, and L. Gor'kov, Sov. Phys. JETP 12, 1243 (1961).

${ }^{19}$ S. Skalski, O. Betbeder-Matibet, and P.R. Weiss, Phys. Rev. 136, 1500 (1963).

20 J.P. Carbotte, M. Greeson, and A. Perez-Gonzalez, Phys. Rev. Lett. 66, 1789 (1991); S.P. Singh, R.K. Pandey, and P. Singh, J. Supercond. 9, 269 (1996).

${ }^{21}$ H. Alloul, P. Mendels, H. Casalta, J.F. Marucco, and J. Arabski, Phys. Rev. Lett. 67, 3140 (1991); T. Miyatake, K. Yamaguchi, T. Takata, N. Koshizuka, and S. Tanaka, Phys. Rev. B 44, 10139 (1991); A.V. Mahajan, H. Alloul, G. Collin, and J.F. Marucco, Phys. Rev. Lett. 72, 3100 (1994); S. Zagoulaev, P. Monod, and J. Jegoudez, Phys. Rev. B 52, 10474 (1995); Physica C 259, 271 (1996); M.B. Maple, C.C. Almasan, C.L. Seaman, S.H. Han, K. Yoshiara, M. Buchgeister, L.M. Paulius, B.W. Lee, D.A. Gajewski, R.F. Jardim, C.R. Fincher, G.B. Blanchet,and R.P. Guertin, J. Supercond. 7, 97 (1994).

22 J. Mesot, and A. Furrer, J. Supercond. (in press); A. Furrer, J. Mesot, P. Allenspach, U. Staub, F. Fauth, and M. Guillaume, in Phase Separation in Cuprate Superconductors, p. 101, E. Sigmund and K.A. Müller Eds., Springer-Verlag, Berlin (1994); O.N. Bakharev, A.V. Dooglav, A.V. Egorov, O.B. Marvin, V.V. Naletov, M.A. Teplov, A.G. Volodin, and D. Wagener, ibid., p. 257; Phys. Rev. B 55, 11839 (1997); J. Supercond. 8, 413 (1995); N. Phillips, R. Fisher, and J. Gordon, in Progress in Low-Temperature Physics 13, D. Brewer Ed. (North-Holland, Amsterdam, 1992), p. 267; J.M. Wade, J.W. Loram, K.A. Mirza, J.R. Cooper, and J.L. Tallon, J. Supercond. 7, 261 (1994); A. Junod, in Studies of High Temperature Superconductors, A. Narlikar Ed. (Nova Science, Commack, NY, 1996).

${ }^{23}$ V.Z. Kresin, Phys. Rev. B 25, 157 (1982).

${ }^{24}$ V.Z. Kresin, Phys. Rev. B 32, 145 (1985).

${ }^{25}$ W. McMillan, Phys. Rev. 175, 537 (1968). 


\section{Figure captions:}

Fig. 1: Temperature dependency (near $T_{c}$ ) of the isotope coeff. $\beta$ a) in the presence of magnetic impurities only, with $T_{c} / T_{c 0} \simeq 0.35$ (solid), 0.5 (dashed line), and $\alpha_{0}=0.025$ (corresponds to $\mathrm{YBa}_{2}\left(\mathrm{Cu}_{1-x} \mathrm{Zn}_{x}\right)_{3} \mathrm{O}_{7-\delta}$ ); b) in the presence of magnetic impurities and non-adiabaticity for $T_{c} / T_{c 0} \simeq 0.5$ (dash-dotted), 0.6 (dotted line). $\alpha_{0}=0.025, \gamma_{s}=0.16$ (corresponds to $\mathrm{Y}_{1-x} \mathrm{Pr}_{x} \mathrm{Ba}_{2} \mathrm{Cu}_{3} \mathrm{O}_{7-\delta}$, see text).

Fig. 2: Isotope coefficient $\tilde{\beta}_{m}$ near $T_{c}$ as a function of magnetic impurity concentration for $T / T_{c}=0.75$ (solid line), 0.85 (dashed) and 0.95 (dotted). $\alpha_{0}=0.025$ (corresponds to the situation of $\left.\mathrm{YBa}_{2}\left(\mathrm{Cu}_{1-x} \mathrm{Zn}_{x}\right)_{3} \mathrm{O}_{7-\delta}\right)$.

Fig. 3a: $\tilde{\beta}_{m+n a}$ as a function of magnetic impurity concentration in the presence of non-adiabaticity for $T / T_{c}=$ 0.75 (solid line), 0.85 (dashed) 0.95 (dotted). $\alpha_{0}=0.025, \gamma=0.16$ and $\tilde{\Gamma}=123 \mathrm{~K}$ (parameters for $\left.\mathrm{Y}_{1-x} \mathrm{Pr}_{x} \mathrm{Ba}_{2} \mathrm{Cu}_{3} \mathrm{O}_{7-\delta}\right)$.

Fig. 3b: Low doping regime of Fig. 3a. In addition, the case without non-adiabaticity is added for comparison (long dashed line, corresponding to $\mathrm{YBa}_{2}\left(\mathrm{Cu}_{1-x} \mathrm{Zn}_{x}\right)_{3} \mathrm{O}_{7-\delta}$ with $\left.T / T_{c}=0.9\right)$.

Fig. 4a: Isotope coeff. $\beta_{m}$ for conventional superconductors at $T=0$ as a function of magnetic impurity concentration for $\Gamma_{2} / \Gamma_{s}=10$ (solid line) and 50 (dashed). $\alpha_{0}=0.3$.

Fig. 4b: Isotope coeff. $\beta_{m}$ for high- $T_{c}$ superconductors at $T=0$ as a function of magnetic impurity concentration for $\Gamma_{2} / \Gamma_{s}=10$ (solid line) and 50 (dashed) $\alpha_{0}=0.025$.

Fig. 5: Isotope coeff. $\beta_{\text {prox }}$ for a proximity system as a function of $t$ for $T / T_{c}=0.1$ (solid line), 0.2 (dashed) and 0.3 (dotted). $\alpha_{0}=0.5$.

Fig. 6: Isotope coeff. $\beta_{\text {prox }}$ for a proximity system as a function of $T / T_{c}$ for $S_{0}=0.2: l=1$ (dashed) $l=0.5$ (solid) and $S_{0}=1: l=1$ (small dots) $l=0.5$ (dotted). $\alpha_{0}=0.5$. 


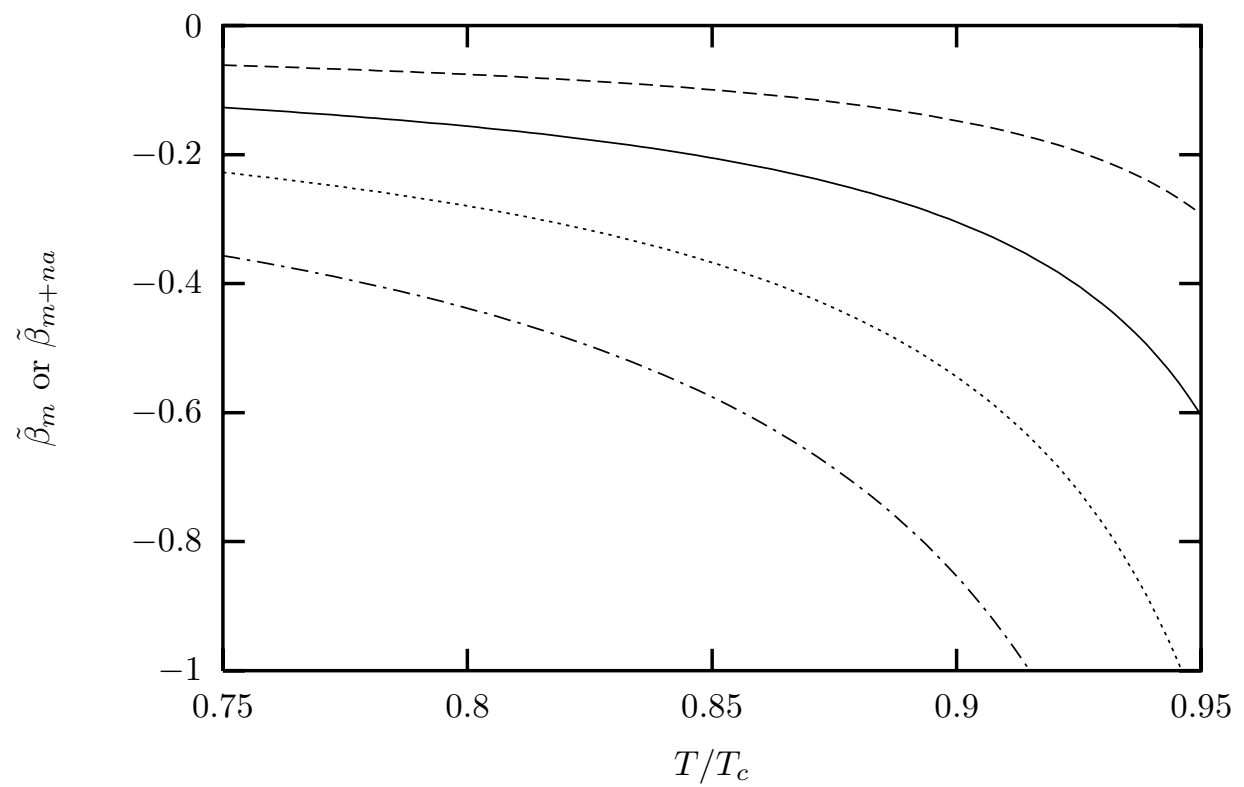

Figure 1

A. Bill et al. 


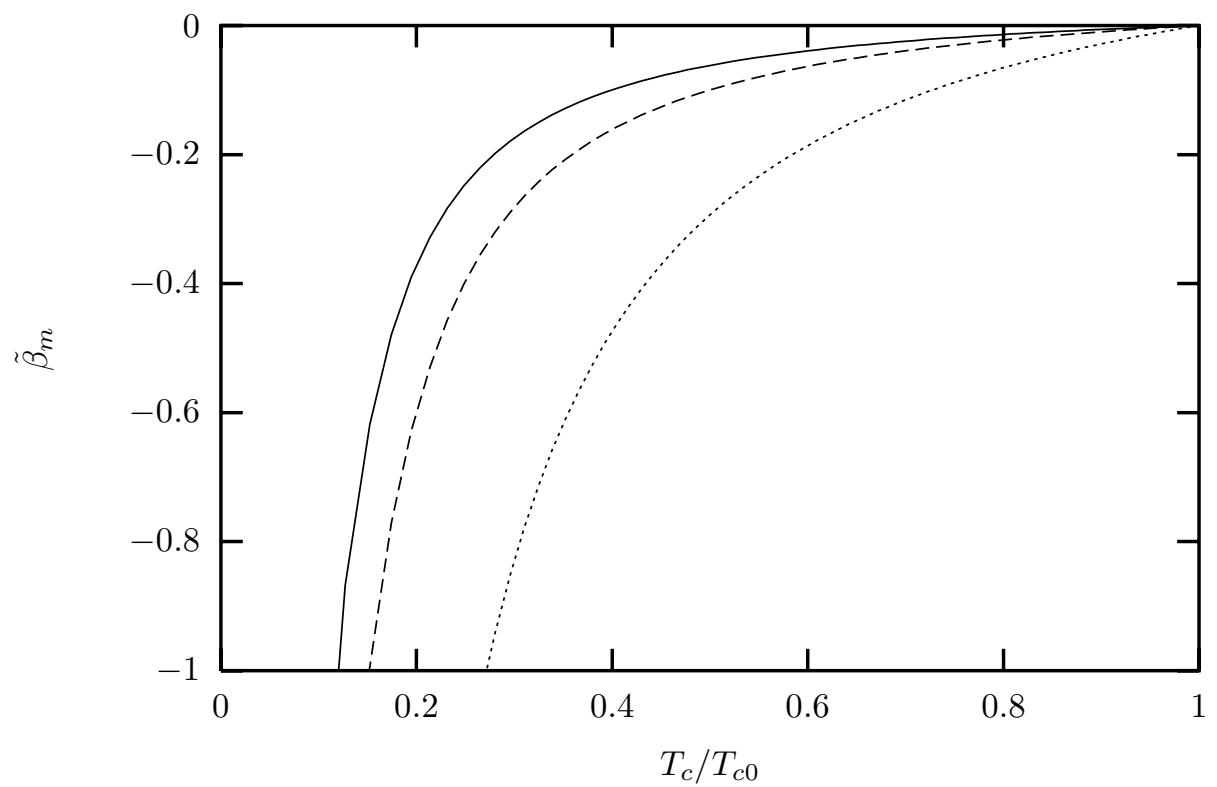

Figure 2

A. Bill et al. 


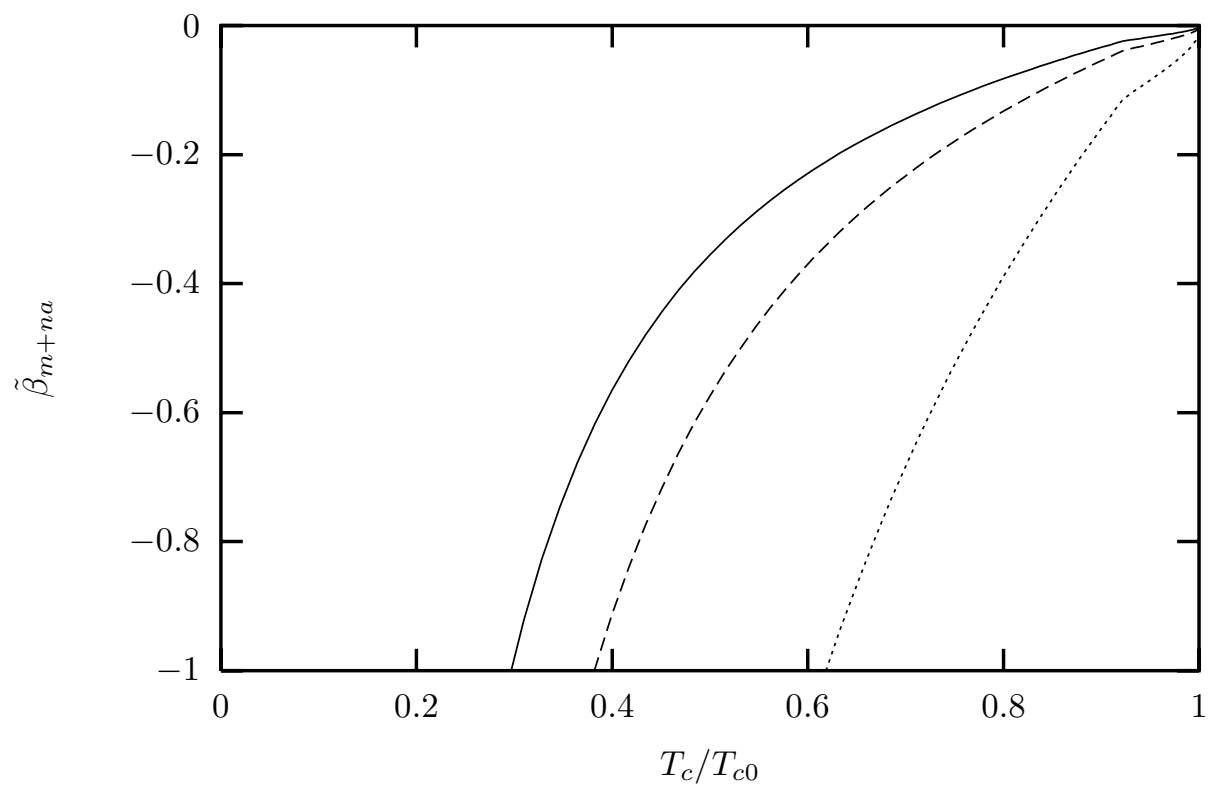

Figure 3a

A. Bill et al. 


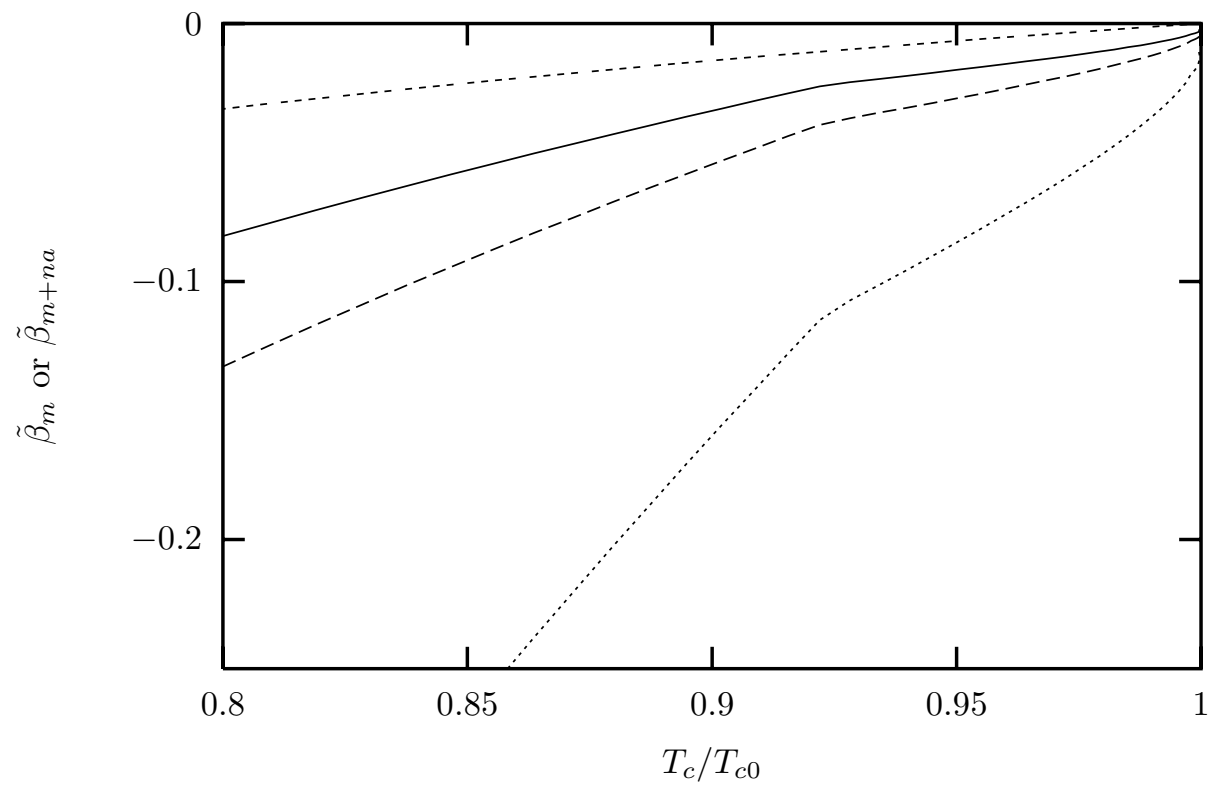

Figure $3 b$

A. Bill et al. 


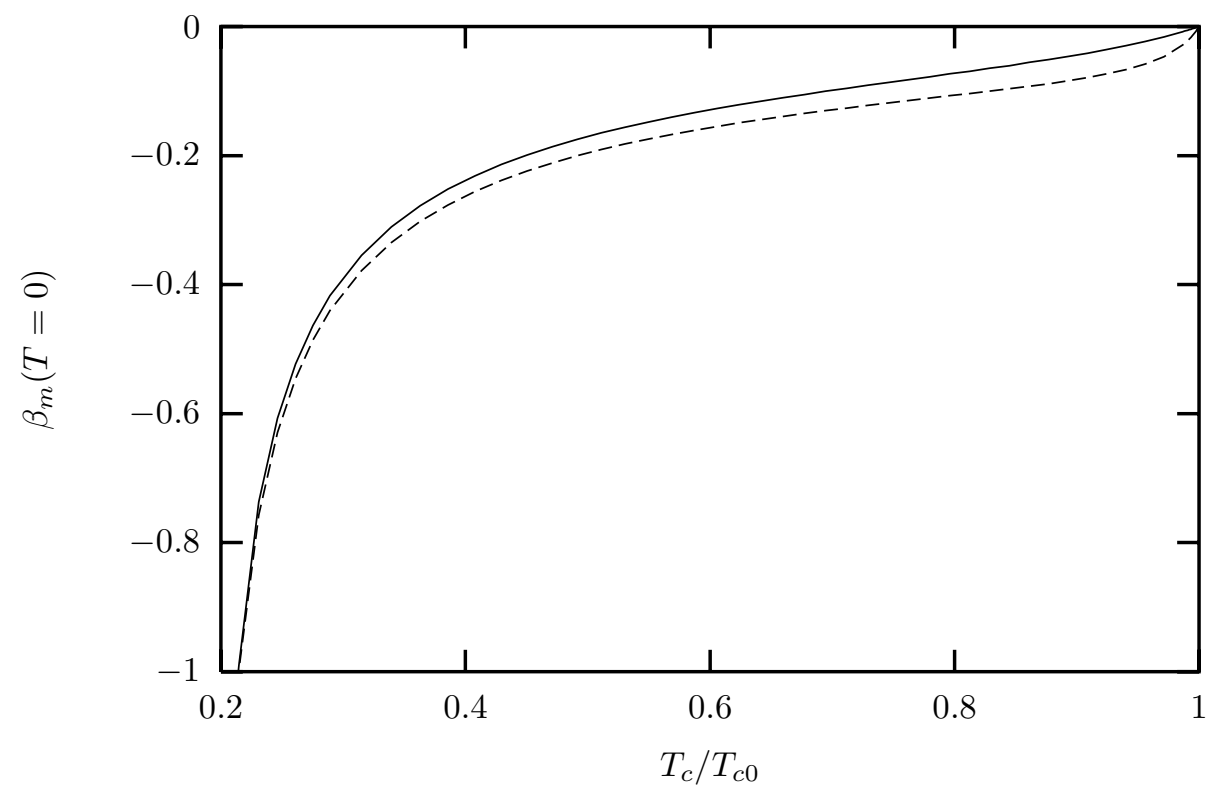

Figure 4a

A. Bill et al. 


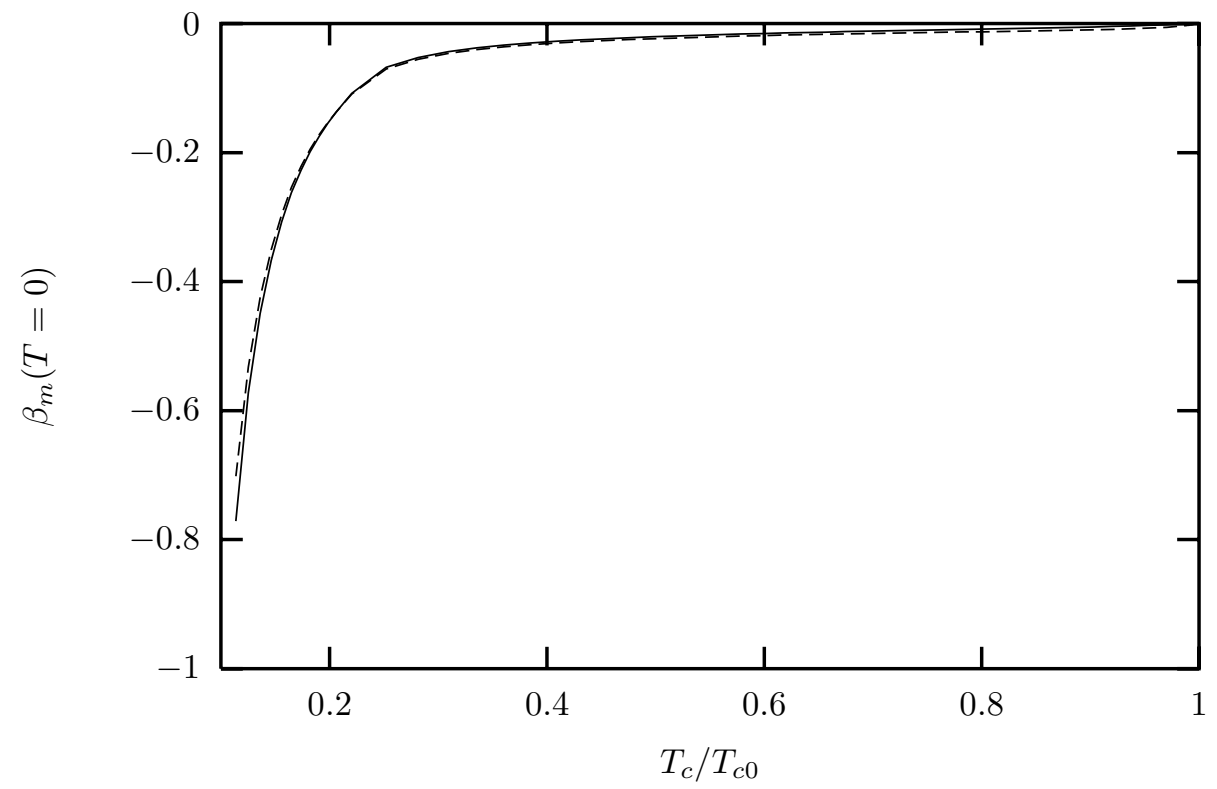

Figure 4b

A. Bill et al. 


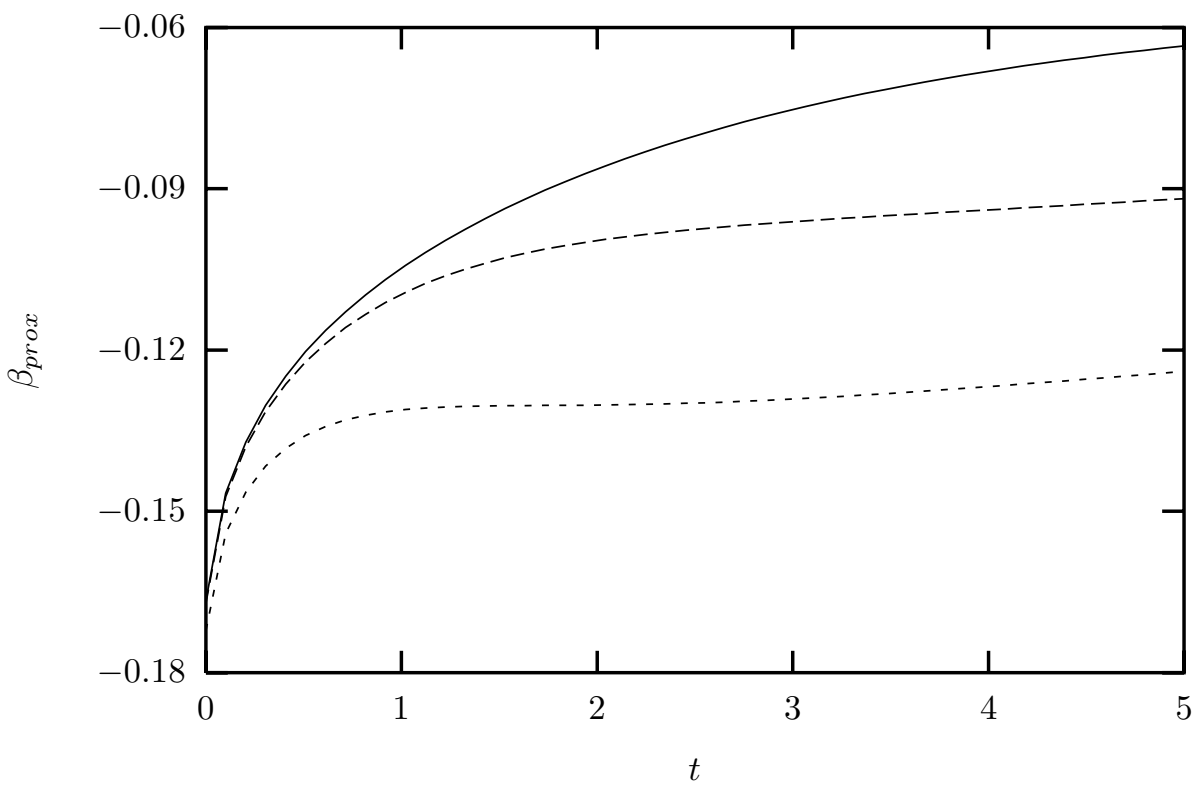

Figure 5

A. Bill et al. 


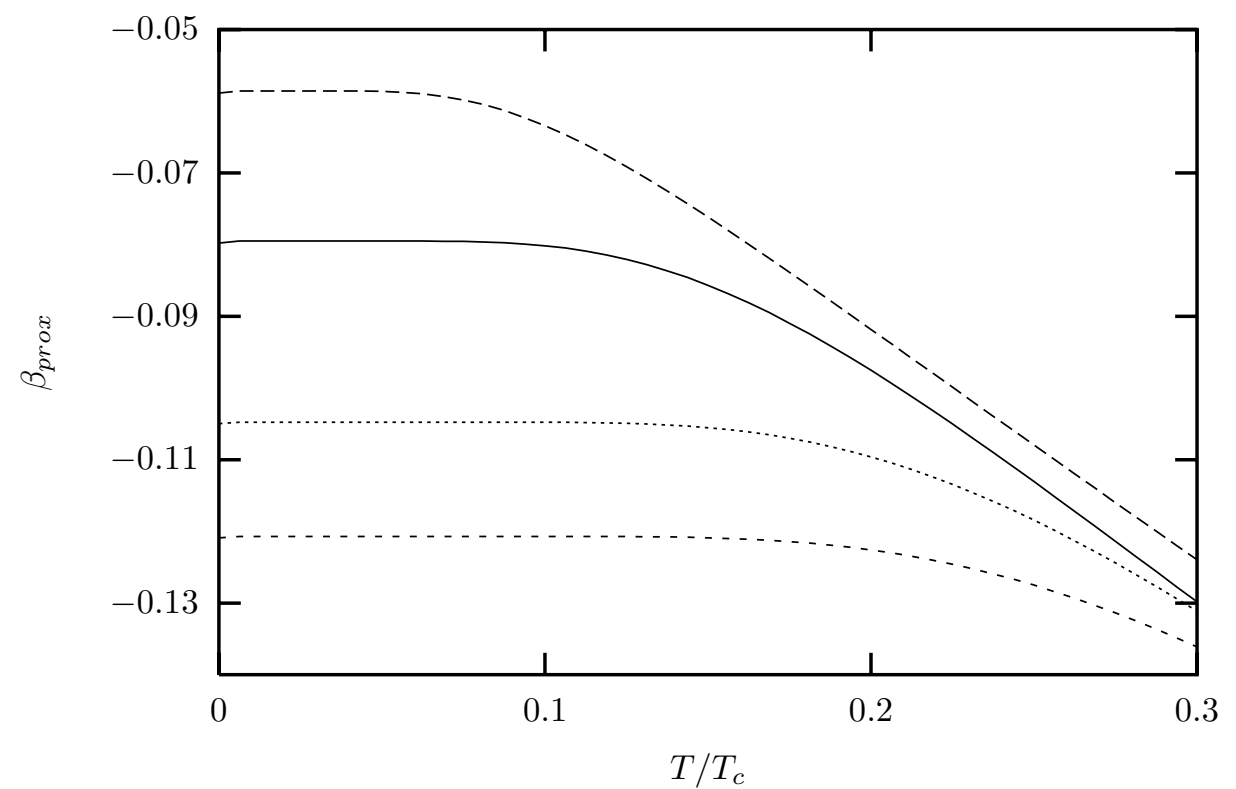

Figure 6

A. Bill et al. 